\title{
Application of thermal analysis in nanotechnology
}

\author{
Carbonization and purification of $\mathrm{nc}^{-\mathrm{TiC}_{x}}$ in argon atmosphere
}

\author{
A. Biedunkiewicz $\cdot$ U. Gabriel $\cdot$ P. Figiel $\cdot$ \\ D. Grzesiak
}

CCTA10 Special Issue

(C) The Author(s) 2010. This article is published with open access at Springerlink.com

\begin{abstract}
The analysis of purification and carbonization process in argon with use of $\mathrm{nc}^{-\mathrm{TiC}_{x}} / \mathrm{C}$ powder, obtained by sol-gel method, is presented. TG-DSC measurements were carried out under non-isothermal and isothermal conditions. The samples were heated up in series to 1473 , 1573, 1673, and $1773 \mathrm{~K}$. For this series description of process kinetics is presented. Four stages of the process have been distinguished. Kinetics was described using Coats-Redfern equation. The kinetic parameters were determined for particular stages. Using obtained kinetic data, the analysis of the process was performed. The $\alpha(T)$ and $r(\alpha, T)$ dependencies on heating rate and temperature were investigated. In order to obtain high carbonization degree and carbide particles of small size, an appropriate temperature and time of process duration have to be determined. Appropriate carbonization and particles size were obtained for series heated up to $1570 \mathrm{~K}$. It has been demonstrated that the oxygen, present at trace level in argon, can react with components of the system in certain range of temperature, influencing the quality of obtained product. The particles have been depicted by TEM method, whereas characterization of structure and particles size was performed by XRD method. MS method was used to determine evolved gaseous products.
\end{abstract}

\footnotetext{
A. Biedunkiewicz $(\bowtie) \cdot$ U. Gabriel · P. Figiel Institute of Materials Science and Engineering, West Pomeranian University of Technology, Szczecin, Av. Piastow 19, 70-310 Szczecin, Poland

e-mail: anna.biedunkiewicz@ zut.edu.pl

D. Grzesiak

Institute of Mechanical Technology, West Pomeranian University of Technology, Szczecin, Av. Piastow 19, 70-310 Szczecin, Poland
}

Keywords Carbonization - Kinetic - Nanomaterials · Purification - Titanium carbide

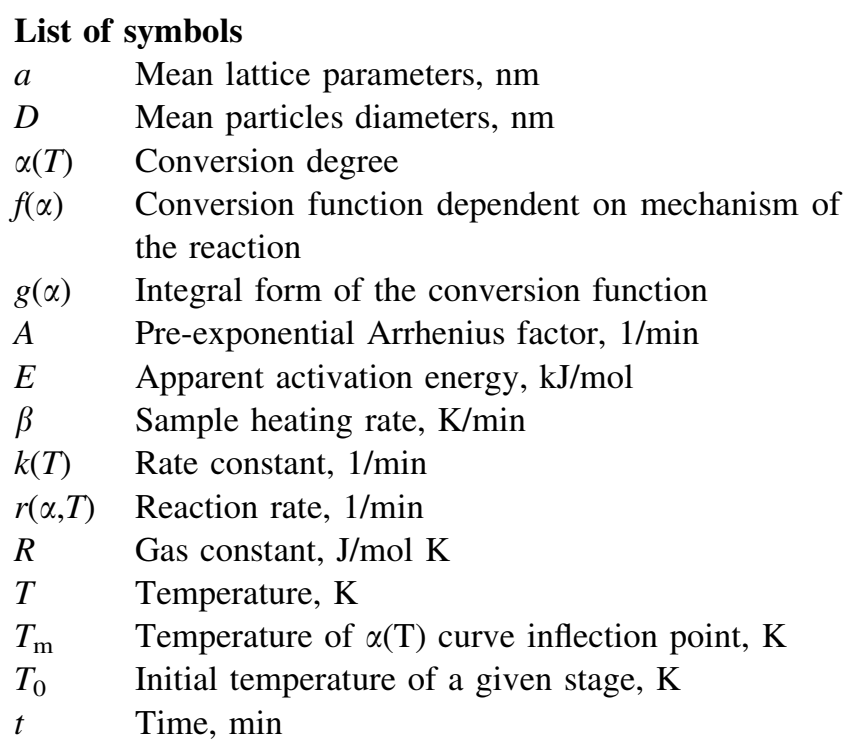

\section{Introduction}

Carbides, nitrides, and borides of transition metals belong to the group of ceramic materials known as conventionally hard materials. This results from the character of chemical bound and crystallographic structure. Nanocrystalline materials, playing the part of one of the phases of nanocomposite, can contribute to the occurrence of strengthening phenomena not existing in conventional materials of micrometric size.

Appropriate selection of investigation methodology enables understanding of process mechanisms, performing 
quantitative analysis, and then correct determination of synthesis conditions. The selection of investigation methodology and the way of determination of nanomaterials synthesis conditions were the purpose of this study.

Available analytical techniques were not sufficient to bring the way of synthesis elaborated by the authoress to manufacturing technology of unambiguously defined final product. The internal randomness of the process, resulting from the nature of nanomaterial, and the external randomness resulting from limitations of analytical techniques indicated necessity of statistical depiction. Complex mechanism of sol-gel synthesis, increased chemical activity of nanoparticles in oxidizing environments, even occurring in trace amounts in high-purity gases, required application of precise methods and performing investigations for a wide range of parameters.

The original way of ceramic nanomaterials synthesis based on non-hydrolytic sol-gel method was elaborated [18]. The method consists in mixing at molecular level solution components which, thanks to the donor-acceptor interactions, get coordinated initializing spatial cross-linking. Proceeding organic-inorganic polymerization fixes the system coordination at the gelling stage. Gels containing carbon, along with donor groups, enable formation of complex gels, in which one component forms organic network enclosing inorganic clusters. This is the first stage of elaborated method. The intermediate product, containing low stoichiometric nanocrystalline carbides, like $\mathrm{TiC}_{x}$ $(x \leq 0.7)$ in carbon matrix, is obtained. In second high temperature stage carbonization and purification of raw material takes place. The process is carried out in argon.

Obtaining the material of high carbonization degree, remaining the right grains size and properties, is essential. Selection of parameters meeting these requirements is difficult. In this study kinetic investigations were applied. Kinetic studies have major significance during investigations of conversions proceeding with use of nanomaterials $[5,8]$. They allow to identify the intermediate and final products, distinguish stages of the process, determine their temperature ranges, and acquire the quantitative description. The base of kinetic description of these processes is usually thermogravimetric measurements TG-DSC.

\section{Experimental}

The kinetic investigations were carried out by thermoanalytical method using TG-DSC (SDT Q600, TA) coupled with MS (Thermostar GDS 301 Pfeiffer Vacuum) for gaseous products identification. Measurements were performed in non-isothermal $(\beta=10,20$, and $50 \mathrm{~K} / \mathrm{min})$ and isothermal $(6 \mathrm{~h})$ conditions. After parameters determination the way of synthesis in larger scale was elaborated.
Performance parameters of ceramic nanocomposite materials manufacturing depend on type of material. The procedure has been presented at the example of nc-TiC carbonization and purification in argon.

Nanoparticles size, lattice parameter, chemical, and phase composition before and after heat-treatment were determined with the following techniques: XRD (Philips PW3040/x0 X'Pert Pro), HRTEM (JEM 3010), SEM (JEOL JSM 6100), EDX (Oxford Instruments, ISIS 300), XPS (SIA 100 Cameca), total carbon measurement (MULTI EA2000, Analytic Jena).

High-purity argon 'Alphagaz $2 \mathrm{Ar}$ ' from Air Liquide $\left(\mathrm{H}_{2} \mathrm{O}<\mathrm{ppm} / \mathrm{mol}, \mathrm{O}_{2}<0.1 \mathrm{ppm} / \mathrm{mol}, \mathrm{C}_{n} \mathrm{H}_{m}<0.1 \mathrm{ppm} /\right.$ $\mathrm{mol}, \mathrm{CO}<0.1 \mathrm{ppm} / \mathrm{mol}, \mathrm{CO}_{2}<0.1 \mathrm{ppm} / \mathrm{mol}, \mathrm{H}_{2}<0.1$ $\mathrm{ppm} / \mathrm{mol}$ ) was used during experiments. With regard to reactivity of $\mathrm{O}_{2}$ the special attention during measurements was given to possibility of oxidation of components of the system during the process run.

During the measurements the samples were heated up to $1473,1573,1673$, and $1773 \mathrm{~K}$. These temperature values correspond to the isothermal conditions. The samples were heated in isothermal conditions for $6 \mathrm{~h}$. Argon purge flow rate during measurements was set at $100 \mathrm{~cm}^{3} / \mathrm{min}$.

Dependency of the reaction rate on temperature was described applying Arrhenius equation. After separation of variables the reaction rate of particular stage in heterogeneous process is expressed as [9-15]:

$\frac{\mathrm{d} \alpha}{\mathrm{d} t}=A \exp \left\{-\frac{E}{R T}\right\} f(\alpha)$

After integration of Eq. 1, assuming constant temperature (isothermal process), the resulting equation takes the form

$g(\alpha)=\int_{0}^{\alpha} \frac{\mathrm{d}(\alpha)}{f(\alpha)}=k(T) \int_{0}^{t} \mathrm{~d} t$

Thus

$g(\alpha)=k(\alpha) \cdot t$

Non-isothermal measurements are usually carried out at constant heating rates $\beta=\frac{\mathrm{d} T}{\mathrm{~d} t}$ for samples in series. Equation 1 for non-isothermal conditions is written therefore in the following form:

$\frac{\mathrm{d} \alpha}{\mathrm{d} T}=\frac{A}{\beta} \exp \left\{-\frac{E}{R T}\right\} f(\alpha)$

After integration of Eq. 4, one obtains

$g(\alpha)=\frac{A}{\beta} \int_{T_{0}}^{T} \exp \left\{-\frac{E}{R T}\right\} \mathrm{d} T$

The integral appearing on the right-hand side of Eq. 5 does not have analytical solution. 
The commonly used approximate solution is the CoatsRedfern equation [16]

$\ln \left(\frac{g(\alpha)}{T^{2}}\right)=\ln \left[\frac{A R}{\beta E}\left(1-\frac{2 R T_{\mathrm{m}}}{E}\right)\right]-\frac{E}{R T}$

In calculations the assumption, that $\left(1-\frac{2 R T_{\mathrm{m}}}{E}\right)$ factor has approximately constant value, is made.

On the basis of experimental data the form of $g(\alpha)$ function (kinetic model) best fitting experimental data, Arrhenius parameters $A$ and $E$ have to be determined.

In case of real processes deviations from linearity occur, errors do not fulfill the normal distribution, and $\ln A$ and $E$ values are strongly linearly correlated. This complicates the calculations to such a degree, that no method is appropriate. It should be added that there were many research works devoted to this issue [10, 17-21].

In isothermal conditions one of the variables (temperature) in series is constant, while conversion degree changes. In non-isothermal conditions both temperature and conversion degree change. It is general opinion that this causes difficulties in separation of $k(T)$ and $f(\alpha)$ or $g(\alpha)$ functions [10]. In our opinion in case of properly functioning measurement system the problem should not exist.

Non-isothermal methods are important, as from single measurement full information about the process can be obtained. There are only needed the methods enabling control at every step of kinetic analysis. It is necessary therefore to estimate directly, in the independent manner, experimental data, i.e., recorded courses of TG, DTG, and HF functions, conversion degrees calculated for the stages, correctness of kinetic models identification, and calculated Arrhenius parameters values. Systems classified to computational intelligence, particularly artificial intelligence methods, posses this property. Artificial neural networks were applied before for nonisothermal measurements analysis in works $[4,6-8,12$, 14, 15].

Using carbonized and purified nc-TiC particles the technology of the production of the nanocomposite structures based on the selective laser melting (SLM) technology was worked out. The MCP HEK Realizer II device was applied. Suitable gear and the modification of the parameters of the laser energy delivering allowed to use the device for work in the Partial Melting technology. Such configuration enables the research on obtainment of the nanocomposite material. As matrix the stainless steel 1.4404 was used and the filler (nanoparticles) was nc$\mathrm{TiC}_{x+y}$. Samples were subjected to hardness tests for quick estimation of the mechanical parameters. Chosen samples with highest hardness were submitted to observations on the scanning microscope.

\section{Results}

In Fig. 1, the results of XRD measurements of the samples after carbonization are presented. Particles' mean diameter was estimated using Scherrer method.

Well-carbonized $\mathrm{nc}^{-\mathrm{TiC}} \sim 0.85$, with average particles diameter in the order of $40 \mathrm{~nm}$, was obtained during heating the samples in temperature of 1520-1573 K. TEM image of the powder after carbonization in $1573 \mathrm{~K}$ is shown in Fig. 2. These grains were 30\% larger comparing to size of the grains before carbonization.

The process parameters were determined basing on investigations by TG-DSC method. In Figs. 3 and 4 plots of normalized TG and HF curves for the used heating rates are presented, respectively.

The first sections of temperature function correspond to non-isothermal conditions, the short, poorly visible

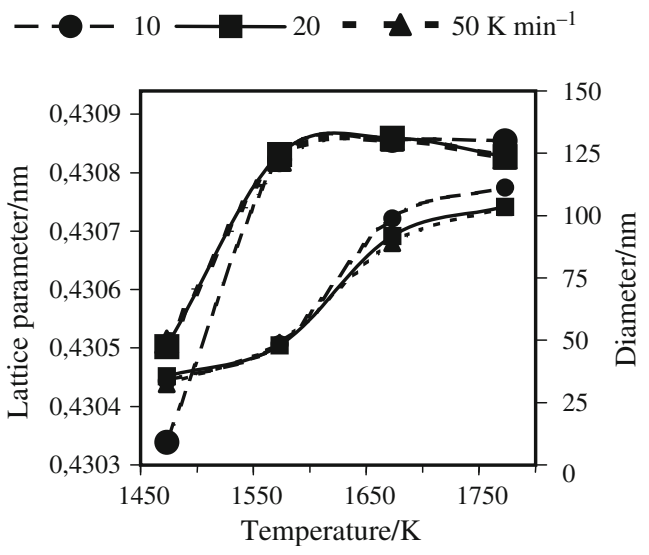

Fig. 1 Dependency of nc-TiC mean lattice parameters $(a)$ and mean particles diameters $(D)$ on temperature

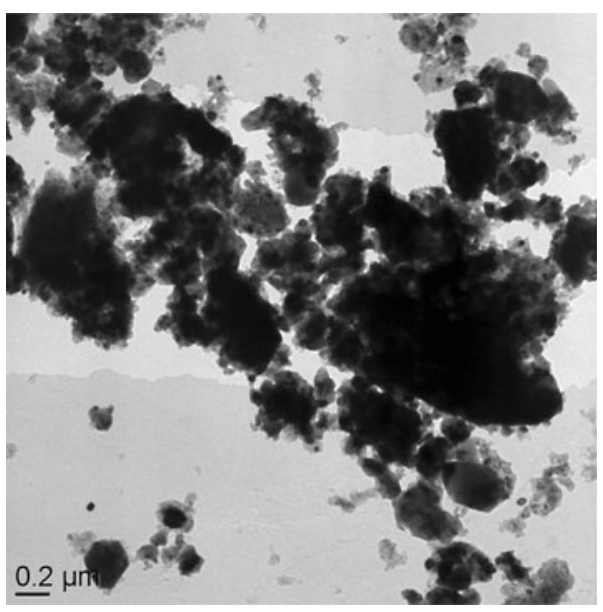

Fig. 2 TEM image of nc-TiC after carbonization 


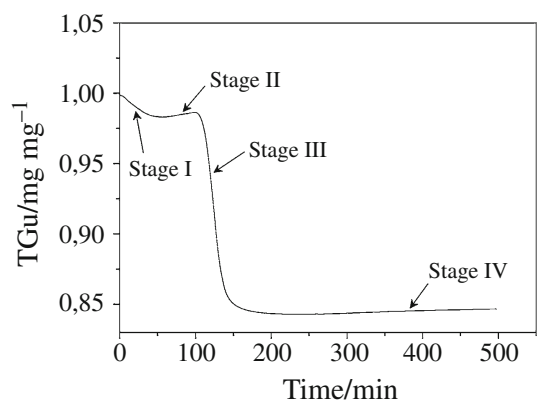

Fig. 3 Plots of normalized TG functions in time for $\beta=10 \mathrm{~K} \cdot \mathrm{min}^{-1}$

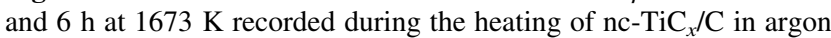
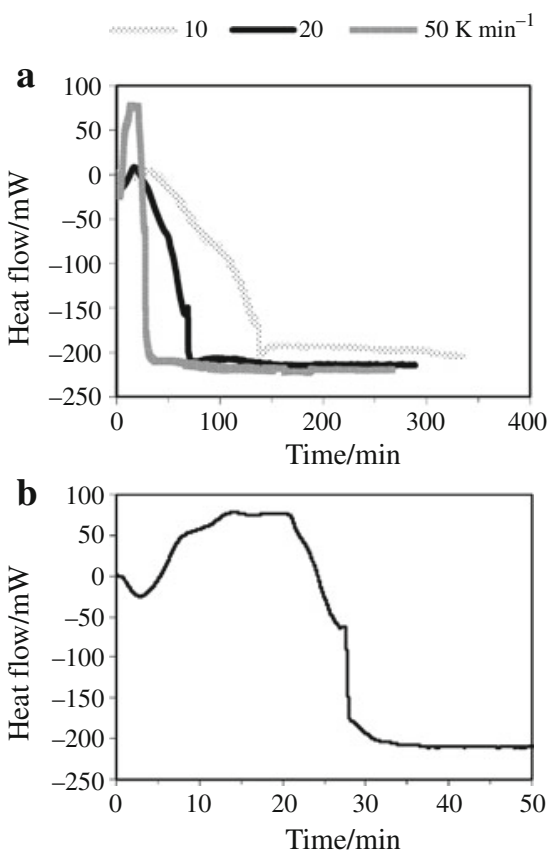

Fig. $4 \mathrm{HF}$ functions in time for (a) $\beta=10,20$, and $50 \mathrm{~K} \cdot \mathrm{min}^{-1}$ and $6 \mathrm{~h}$ at $1673 \mathrm{~K}$ (b) $\beta=50 \mathrm{~K} \cdot \mathrm{min}^{-1}$, recorded during the heating of $\mathrm{nc}-\mathrm{TiC}_{x} / \mathrm{C}$ in argon, respectively

segments correspond to the transient regime, and the straight lines to isothermal conditions.

Based on the TG curves four stages have been distinguished (Fig. 3). The first, endothermic stage proceeding with mass loss, corresponded to desorption of volatile products, D3 model. The second, exothermic stage proceeding with mass growth, was assigned to oxidation of uncarbonized $\mathrm{nc}^{-}-\mathrm{TiC}_{x} / \mathrm{C}$ by the oxygen present in argon at trace level, F2 model. The third endothermic stage, proceeding with mass loss, referred to carbonization of nc$\mathrm{TiC}_{x} / \mathrm{C}$ and pyrolysis of organic compounds, contained in the raw samples. The pyrolysis of admixtures and car-

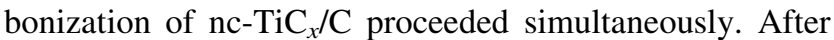
completing the carbonization process at the temperature above $1573 \mathrm{~K}$, oxidation of carbonized $\mathrm{TiC}_{x+y}$ by oxygen
Table 1 List of indentified kinetic models

\begin{tabular}{lll}
\hline Model & $g(\alpha)$ & Stage \\
\hline D3 & $\frac{3}{2}\left[1-(1-\alpha)^{\frac{1}{3}}\right]^{2}$ & I \\
F2 & $(1-\alpha)^{-1}-1$ & II \\
F1 & $-\ln (1-\alpha)$ & III \\
D3 & $\frac{3}{2}\left[1-(1-\alpha)^{\frac{1}{3}}\right]^{2}$ & \\
R2 & $2\left[1-(1-\alpha)^{\frac{1}{2}}\right]^{2}$ & IV \\
\hline
\end{tabular}

present in argon at trace level was observed, R2 model. The indentified kinetic models were listed in Table 1.

The third, basic stage preceded in non-isothermal and isothermal conditions; at lower conversion degrees F1 model (first-order reaction) and at the higher conversion degrees (above 0.98) D3 model (three-dimensional diffusion, spherical symmetry, Jander equation) was applied.

The course of reaction was evaluated on the basis of value of reaction rate constant calculated according to formula (3). Results of calculations are presented in Fig. 5.

The values of $\alpha(T)$ calculated for the given sample heating rate from the Coats-Redfern equation were used. The obtained results for the third stage are shown in Fig. 6.

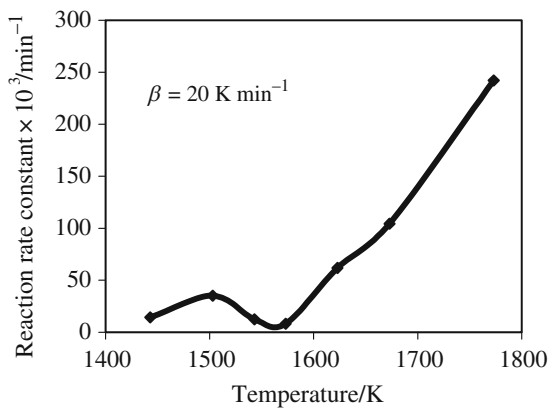

Fig. 5 Dependency of reaction rate constant on temperature. F1 model

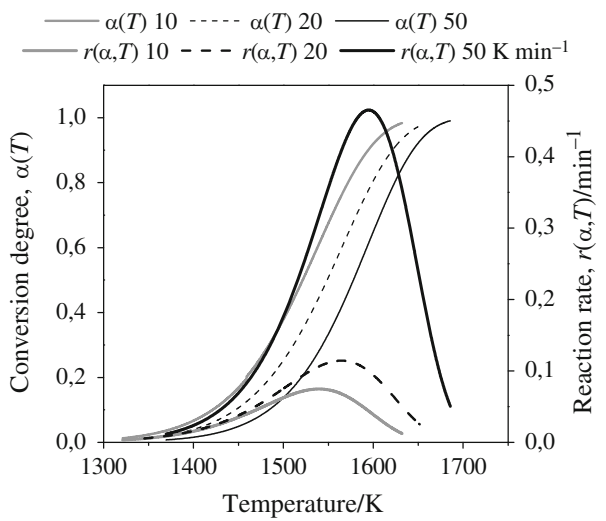

Fig. 6 Plots of $\alpha(T)$ and $r(\alpha, T)$ functions. Purification and carbonization of $\mathrm{nc}-\mathrm{TiC} / \mathrm{C}$ in argon. Third stage, model F1 
The $\alpha(T)$ and $r(\alpha, T)$ plots for stage do not come to an end, because at high conversion degrees process ran according to the different kinetic models.

The determined dependencies are in accordance with the theory. With the increase in sample heating rates $\alpha(T)$ curves are shifted into the higher temperature range. The process rate increases from zero for $\alpha(T)$ equal zero, reaches the maximum in temperature $T_{\mathrm{m}}$, and then usually decreases to zero at $\alpha(T) \rightarrow 1$. The temperature ranges for stages run are consistent with the ones determined experimentally.

In considered range of temperature two processes preceded simultaneously; pyrolysis of organic compounds, contained in the raw samples and proceeding with their

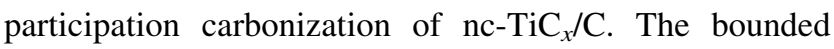
carbon remains in the system. As a result the lesser sample mass losses were observed. In lower temperature proceeds also pyrolysis. Carbonization starts in temperature of about $1480 \mathrm{~K}$ and becomes a dominating process in temperature of about $1570 \mathrm{~K}$. At $1610 \mathrm{~K}$ pyrolysis becomes a dominating process again. With regard to carbonization effectiveness, the process should be carried out in temperature of about $1570 \mathrm{~K}$. The minimum on the curve $k(T)$ has been observed (Fig. 5).

Carbonization of $\mathrm{nc}-\mathrm{TiC} \sim 0.7$ proceeded well in temperature of about $1570 \mathrm{~K}$. Heating time in isothermal conditions was experimentally established. It equaled about $6 \mathrm{~h}$. Extended heating process gave larger crystals but stoichiometric rate C:Ti was unchanged. Diffractograms of nc-TiC sample before and after purification in argon are shown in Fig. 7. After purification carbonized nc-TiC $\sim 0.85$ without carbon matrix was observed.

Composite materials obtained by SLM technique, containing nc-TiC after carbonization and purification in steel matrix, were characterized by good dispersion of $\mathrm{TiC}$ nanoparticles in matrix, and three times higher hardness

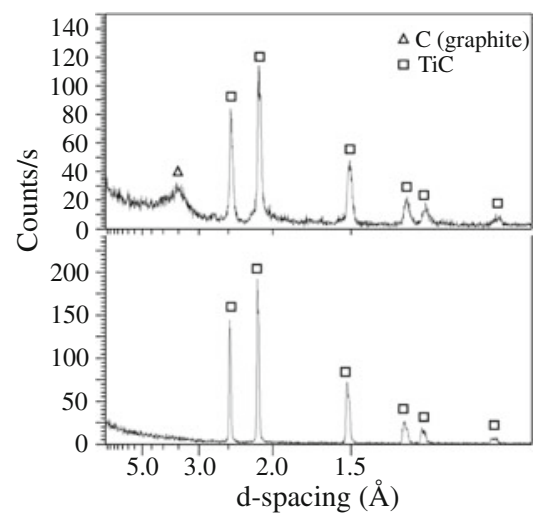

Fig. 7 X-ray diffractogram of nc-TiC in carbon matrix before (top) and after purification (bottom)

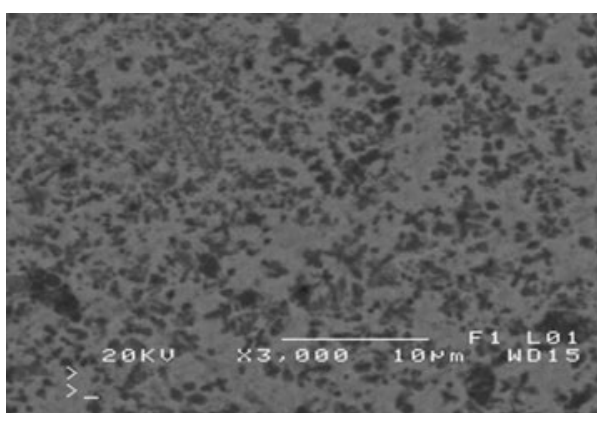

Fig. 8 SEM image of composite of purified nc-TiC $\sim 0.85$ in steel matrix

than stainless steel. SEM image of the composite is presented in Fig. 8.

\section{Conclusions}

The significant input into nanotechnology is made by elaborated system of analysis of processes results, consisting in application of artificial neural networks, and in case of kinetic investigations also adaptation of theory of heterogeneous processes chemical kinetics for description of processes proceeding with participation of nanomaterials.

The elaborated method of nanocrystalline titanium carbides synthesis belongs to sol-gel technique. The process consists of two stages. In the first stage low-temperature synthesis of nanocrystallines proceeds in situ in carbon matrix. In the second stage of the process, elimination of carbon from the matrix and carbonization of $\mathrm{TiC}_{x}$ take place. The advantage of this technology is possibility of control of nanoparticles formation and growth. It consists in physical limitation of volume available for the growth of single nanoparticle by use of carbon matrix. Properly designed process enables to anticipate chemical composition and size of nanoparticles and program in a controlled way their coalescence and growth to appropriate size.

The bases were kinetic investigations. The procedure was presented at the example of nc-TiC synthesis. The course of the process was investigated by TG-DSC method in argon of 6.0 purity class, in non-isothermal and isothermal conditions, at different samples' heating rates. Carbonization degree and crystallites size of the $\mathrm{nc}^{-\mathrm{TiC}_{x}}$ were determined by TC, XRD, and TEM methods. The formed gaseous products were also identified by MS method.

The four stages of the process were distinguished. In the first stage evolution and desorption of volatile products contained in the raw samples took place. In the second stage the uncarbonized $\mathrm{nc}-\mathrm{TiC}_{x}$ was partially oxidized by the oxygen present in argon at trace level. In the third stage the pyrolysis of organic admixtures and carbonization of 
nc-TiC $\mathrm{C}_{x}$ proceeded simultaneously. Carbonization of nc$\mathrm{TiC}_{x}$ proceeded well in temperature of about $1570 \mathrm{~K}$. Heating time in isothermal conditions equaled about $6 \mathrm{~h}$. Good dispersion of titanium carbide in steel matrix and significant increase hardness were obtained using purified, high stoichiometry nc-TiC $\sim 0.85$ for nano-composite manufacturing by SLM method.

Acknowledgements This article and the work it concerns were partially generated in the context of the MULTIPROTECT project, funded by the European Community as contract No. NMP3-CT-2005011783 under the 6th Framework Programme for Research and Technological Development. Financial support of part of the work by the Ministry of Science and Higher Education within the Project No. N N507 444334, 2008-2011, is gratefully acknowledged.

Open Access This article is distributed under the terms of the Creative Commons Attribution Noncommercial License which permits any noncommercial use, distribution, and reproduction in any medium, provided the original author(s) and source are credited.

\section{References}

1. Biedunkiewicz A, Wysiecki M, Noworol P. Organotitanium precursor and method of producing and processing of organotitanium precursor. Polish Patent P200978 (2008).

2. Biedunkiewicz A, Wysiecki M, Jasiński W. Synthesis of titanium-carbon coatings via process of liquid phase coatings (LPC). In: Proc. Eur. Conf. Advanced Materials and Applications, Maastricht, vol. 3 ; 1997. p. 177-80.

3. Biedunkiewicz A, Mozdzen G, Strzelczak A. Assessment of hightemperature corrosion resitance of Ti-Si-C-N nanocomposites in dry air. In: Proc. Eur. Congress EUROMAT 2007 on Advanced Materials and Processes, Nürnberg, Germany; 2007.

4. Biedunkiewicz A, Strzelczak A, Mozdzen G, Lelatko J. Nonisothermal oxidation of ceramic nanocomposites using the example of Ti-Si-C-N powder: kinetic analysis method. Acta Mater. 2008;56:3132-45.

5. Biedunkiewicz A, Jasinski W, Lenart S. Synthesis and growth of Ti-C coatings from the sol-gel process. Vacuum. 1998;50(1-2): $65-8$.
6. Biedunkiewicz A, Figiel P, Jedrzejewski R. Effect of Heat Treatment on $\mathrm{TiC}_{\mathrm{x}}-\mathrm{C}$ Ceramic Nanocomposites. In: Proc. Int. Conference on Advanced Processing ofr Novel Functional Materials-APNFM 2008, Dresden, Germany; 2008. p. 132.

7. Biedunkiewicz A. Purification and carbonization of $\mathrm{nc}-\mathrm{TiC} / \mathrm{C}$ in argon. Mater Eng (Polish). 2008;6:663-73.

8. Biedunkiewicz A, Gordon N, Straszko J, Tamir S. Kinetics of thermal oxidation of titanium carbide and its carbon nano-composites in dry air atmosphere. J Therm Anal. 2007;3:717-22.

9. Straszko J, Olszak-Humienik M, Możejko J. Kinetics of thermal decomposition of the solid state. Eng Chem Proc (Polish). 1995; $1: 45-9$.

10. Vyazovkin S, Wigth ChA. Model-free and model-fitting approaches to kinetic analysis of isothermal and nonisothermal data. Thermochim Acta. 1999;340-341:63-8.

11. Baitalow F, Smith HG, Wolf G. Formal kinetic analysis of processes in the solid state. Thermochim Acta. 1999;337:111-20.

12. Strzelczak A. Analysis of the oxidation processes selected nanocomposites in dry air. Thesis, SUT, Szczecin; 2008.

13. Straszko J, Olszak-Humienik M, Możejko J. Study of The Mechanism and Kinetic Parameters of The Thermal Decomposition of Cobalt Sulphate Hexahydrate Thermal. Therm Anal Calorim. 2000;59:935-42.

14. Biedunkiewicz A, Chrościechowska J, Strzelczak A. Non-isothermal oxidation of TiCx powder in dry air. Pol J Chem Technol. 2005;7(4):1-10.

15. Straszko J, Biedunkiewicz A, Strzelczak A. Application of artificial neural networks in oxidation kinetic analysis of nanocomposites. Pol J Chem Technol. 2008;10(3):21-8.

16. Costs AW, Redfern JP. Kinetic parameters from thermogravimetric data. Nature. 1964;201:68-9.

17. Brunaer M, Shachom M. Statistical analysis of linear and nonlinear correlation of the Arrhenius equation constant. Chem Eng Proc. 1997;36:248-9.

18. Borah D, Baruah MK. Proposed novel dynamic equations for direct determination of activation energy in non-isothermal isothermal systems. Fuel Proc Technol. 2005;86:781-94.

19. Schwaab M, Pinto JCh. Optimum reference temperature for reparameterization of the Arrhenius equation. Part 1. Problems involving one kinetic constant. Chem Eng Sci. 2007;68:2750-64.

20. Lyon RE. An integral method of nonisothermal kinetic analysis. Thermochim Acta. 1997;297:117-24.

21. Sbirrazzuoli N, Vincent L. Comparison of several computational procedures for evaluating the kinetics of thermally stimulated condensed phase reactions. Chem Intell Lab Syst. 2000;54:53-60. 